\title{
Psychiatric symptoms and their association with sleep disturbances in intensive care unit survivors
}

This article was published in the following Dove Medical Press journal: International Journal of General Medicine

\section{Sophia Wang, ${ }^{1-3}$ Jared W Meeker, ${ }^{4}$ Anthony J Perkins, ${ }^{5}$ Sujuan Gao, ${ }^{5}$ Sikandar H Khan, ${ }^{4,6}$ Ninotchka L Sigua, ${ }^{4,7}$ Shalini Manchanda, ${ }^{4,7}$ Malaz A Boustani, ${ }^{2,3,6,8}$ Babar A Khan $^{3,4,6,8}$}

'Department of Psychiatry, Indiana University School of Medicine, Indianapolis, IN 46202 USA;

${ }^{2}$ Center for Health Innovation and Implementation Science, Clinical and Translational Science Institute, Indianapolis, IN, USA; ${ }^{3}$ Sandra Eskenazi Center for Brain Care Innovation, Eskenazi Hospital, Indianapolis, IN, USA; ${ }^{4}$ Division of Pulmonary, Critical Care, Sleep and Occupational Medicine, Department of Medicine, Indiana University School of Medicine, Indianapolis, IN, USA; ${ }^{5}$ Department of Biostatistics, Indiana University School of Medicine, Indianapolis, IN, USA; 'IU Center of Aging Research, Regenstrief Institute, Indianapolis, IN, USA; ${ }^{7}$ Indiana University Health Sleep Disorders Center, Indiana University School of Medicine, Indianapolis, IN, USA; ${ }^{8}$ Division of Geriatrics and General Internal Medicine, Department of Internal Medicine, Indiana University School of Medicine. Indianapolis, IN, USA

Correspondence: Sophia Wang Department of Psychiatry, Indiana University School of Medicine, $355 \mathrm{~W}$ I6th Street, Suite 4800 GH, Room 4250, Indianapolis, IN 46202, USA

$\mathrm{Tel}+\mathrm{I} 3179637288$

Fax +I 3179637313

Email sophwang@iupui.edu
Background: Sleep disturbances in critically ill patients are associated with poorer long-term clinical outcomes and quality of life. Studies are needed to better characterize associations and risk factors for persistent sleep disturbances after intensive care unit (ICU) discharge. Psychiatric disorders are frequently associated with sleep disturbances, but the role of psychiatric symptoms in sleep disturbances in ICU survivors has not been well-studied.

Objective: To examine the association between psychiatric symptoms and sleep disturbances in ICU survivors.

Methods: 112 adult ICU survivors seen from July 2011 to August 2016 in the Critical Care Recovery Center, an ICU survivor clinic at the Eskenazi Hospital in Indianapolis, IN, USA, were assessed for sleep disturbances (insomnia, hypersomnia, difficulty with sleep onset, difficulty with sleep maintenance, and excessive daytime sleepiness) and psychiatric symptoms (traumarelated symptoms and moderate to severe depressive symptoms) 3 months after ICU discharge. A multivariate logistic regression model was performed to examine the association between psychiatric symptoms and sleep disturbances. Analyses were controlled for age, hypertension, history of depression, and respiratory failure.

Results: ICU survivors with both trauma-related and depression symptoms (OR 16.66, 95\% CI 2.89-96.00) and trauma-related symptoms alone (OR 4.59, 95\% CI 1.11-18.88) had a higher likelihood of sleep disturbances. Depression symptoms alone were no longer significantly associated with sleep disturbances when analysis was controlled for trauma-related symptoms. Conclusion: Trauma-related symptoms and trauma-related plus moderate to severe depressive symptoms were associated with a higher likelihood of sleep disturbances. Future studies are needed to determine whether psychiatric symptoms are associated with objective changes on polysomnography and actigraphy and whether adequate treatment of psychiatric symptoms can improve sleep disturbances.

Keywords: sleep disturbance, post-traumatic stress disorder, depression, ICU survivor, psychiatric disorders, post-intensive care syndrome

\section{Introduction}

Sleep disturbance is common among intensive care unit (ICU) patients and has a negative impact on a variety of outcomes. ${ }^{1-3}$ More than half of ICU survivors report having insomnia, nightmares, and poor-quality sleep. These symptoms can persist for as long as 12 months after hospital discharge and decrease quality of life. ${ }^{4,5}$ Sleep is crucial for rest, recovery, and well-being. ${ }^{6,7} \mathrm{~A}$ wide spectrum of potential risk factors for post-ICU sleep disturbances have been identified, including hypnotic drug use, illness severity, female gender, older age, respiratory failure, and others. ${ }^{4}$ Previous studies have shown 
an association between psychiatric symptoms and post-ICU sleep disturbances. ${ }^{8-13}$ Sleep deprivation has been linked to delirium, cardiovascular, immune and endocrine dysfunction, cognitive impairment, and even mortality, ${ }^{14-18}$ making identification of risk factors important. The aim of this study was to gain further insight into the relationship between psychiatric symptoms and sleep disturbances in ICU survivors.

We hypothesized that psychiatric symptoms would be associated with post-ICU sleep disturbances in patients recruited from the Critical Care Recovery Center (CCRC) at Eskenazi Hospital in Indianapolis, IN, USA.

\section{Study population}

Two hundred and thirty-three ICU survivors were seen in the CCRC, an ICU survivor clinic, from July 2011 to August 2016. Patients were excluded if they did not complete questions about sleep changes $(\mathrm{N}=49)$, trauma $(\mathrm{N}=43)$, or depression $(\mathrm{N}=29)$. The final sample included 112 patients. Referral criteria to the CCRC were age 18 years or older, admitted to the ICU, on mechanical ventilation or delirious for $\geq 48$ hours (major risk factors for the development of post-intensive care syndrome), ${ }^{19,20}$ and recommended for follow-up by a critical care physician. Exclusion criteria included enrollment into hospice or palliative care services. Indiana University Institutional Review Board (IRB) approval was obtained to conduct retrospective analyses of de-identified clinical data. Medical history and medication lists were collected from patients, informal caregivers, and electronic medical records. Patient consent to review their medical data was not required by the IRB due to anonymization, and the study complied with the Declaration of Helsinki.

\section{Data collection}

Electronic medical records were reviewed to determine whether patients were on an antidepressant, a benzodiazepine, or nonbenzodiazepine hypnotic (zolpidem, zopiclone, eszopiclone, or zaleplon) at their initial CCRC visit (Table 1).

\section{Sleep disturbances}

Patients indicated whether they had any of the following current sleep disturbances (they could mark more than one) as part of an initial CCRC patient assessment 3 months after ICU discharge: insomnia (subjective loss of sleep, nearly every day for two weeks); hard time falling asleep; hypersomnia (subjective excess sleep, nearly every day for two weeks); hard time staying asleep; excessive day time sleepiness (nearly every day for two weeks); and not feeling refreshed in the morning.

\section{Psychiatric symptoms}

Patients completed a depression screening (either the Patient Health Questionnaire-9 (PHQ-9) or Geriatric Depression scale-30 (GDS-30). Patients with PHQ-9 score $\geq 10$ or GDS score $\geq 20$ were categorized as having moderate to severe depressive symptoms. All patients met criterion A of posttraumatic stress disorder (PTSD) from the Diagnostic and Statistical Manual of Mental Disorders (DSM-5)22: exposure to actual or threatened death, serious injury, or sexual violence by direct exposure to threatened death from their ICU stay (ie, critical illness requiring mechanical ventilation for $\geq 24$ hours) and/or delirium, a neuropsychiatric morbidity with mortality rate over twice that of critically ill patients without delirium. ${ }^{21}$ In addition, all patients reported whether they had specific trauma- and stressor-related symptoms which correlated with the DSM-5 criteria for PTSD. ${ }^{22}$ These included criterion B: presence of one (or more) intrusion symptoms associated with the traumatic event(s), beginning after the traumatic event(s) occurred (nightmares? flashbacks or stressful memories of your ICU stay?); criterion $\mathrm{C}$ : persistent avoidance of stimuli associated with the traumatic event(s), beginning after the traumatic event(s) occurred (fear of places and situations which remind me of the ICU); and criterion E: marked alterations in arousal and reactivity associated with the traumatic event(s), beginning or worsening after the traumatic event(s) occurred (jumpiness, I am easily frightened by sudden sounds or sudden movements). ${ }^{22}$

\section{Statistical analyses}

Demographic and clinical characteristics were compared using Fisher's exact tests for categorical variables and Wilcoxon Rank-Sum tests for continuous outcomes (Table 1). A multivariate logistic regression model was performed to examine whether there was an association between psychiatric symptoms and the reported sleep disturbances. Demographic and clinical variables with $P<0.1$ in a univariate analysis (Table 1) and associations with sleep outcomes based on prior research or clinical judgment were included as covariates in a multivariate logistic regression model (Table 2 ). ${ }^{23} P<0.05$ was considered statistically significant. All analyses were performed using SAS v 9.4.

\section{Results}

The study population of 112 ICU survivors with a mean age of 52.2 years was critically ill; $84.8 \%(\mathrm{~N}=95)$ had respiratory failure; and $77.7 \%(\mathrm{~N}=87)$ had delirium. Over one-third $(34.8 \%, N=39)$ had current depressive symptoms, and almost 
Table I Demographics and clinical characteristics of I I 2 ICU survivors grouped by subjective sleep disturbances at the initial CCRC visit

\begin{tabular}{|c|c|c|c|}
\hline Characteristics & $\begin{array}{l}\text { No sleep } \\
\text { disturbances } \\
(\mathrm{N}=53)\end{array}$ & \begin{tabular}{|l} 
Sleep \\
disturbances \\
$(\mathbf{N}=59)$ \\
\end{tabular} & $P$-value \\
\hline \multicolumn{4}{|l|}{ Demographics } \\
\hline Age (years) & $56.8(10.9)$ & $48.0(14.1)$ & 0.001 \\
\hline Female, \% & $45.3(24)$ & $54.2(32)$ & 0.449 \\
\hline African-American, $\%$ & $43.4(23)$ & $42.4(25)$ & 1.000 \\
\hline Education (years) & $11.9(2.4)$ & II.6 (3.2) & 0.513 \\
\hline \multicolumn{4}{|l|}{ Comorbidities } \\
\hline Alcohol use disorder (current or previous), \% & $39.6(19)$ & $24.1(14)$ & 0.097 \\
\hline Tobacco use disorder (current or previous), \% & $8 \mathrm{I} . \mathrm{I}(43)$ & $71.2(42)$ & 0.271 \\
\hline CNS disorder, \% & $45.3(24)$ & $57.6(34)$ & 0.256 \\
\hline Cardiac disease, $\%$ & $4 I .5(22)$ & $28.8(17)$ & 0.171 \\
\hline Hypertension, \% & $77.4(4 I)$ & $59.3(35)$ & 0.046 \\
\hline Diabetes, \% & $30.2(16)$ & $32.2(19)$ & 0.841 \\
\hline COPD, $\%$ & $58.5(3 \mathrm{I})$ & $42.4(25)$ & 0.130 \\
\hline Cancer, \% & I5.I (8) & $17.0(10)$ & 1.000 \\
\hline Psychiatric disorder, \% & $1 \mathrm{II} .3(6)$ & $15.2(9)$ & 0.590 \\
\hline Sleep disorder, \% & $7.6(4)$ & $6.8(4)$ & 1.000 \\
\hline \multicolumn{4}{|l|}{ Hospital characteristics $^{b}$} \\
\hline Length of hospitalization (days) & $17.0(11.3)$ & $21.3(23.3)$ & 0.987 \\
\hline Length of ICU (days) & $12.2(9.8)$ & I3.1 (18.3) & $0.27 \mid$ \\
\hline Delirium during entire hospitalization, \% & $84.9(45)$ & $71.2(42)$ & 0.112 \\
\hline Respiratory failure, $\%$ & $96.2(5 \mathrm{I})$ & $74.6(44)$ & 0.001 \\
\hline \multicolumn{4}{|l|}{ Initial CCRC visit information } \\
\hline Time between initial visit in CCRC and discharge from the hospital (days) & II $6.8(87.2)$ & $93.8(62.3)$ & 0.186 \\
\hline MMSE (0-30 points) & $26.2(4.2)$ & $25.5(4.7)$ & 0.588 \\
\hline Antidepressant prescription at initial CCRC visit, \% & $24.5(13)$ & $42.4(25)$ & 0.071 \\
\hline Benzodiazepine or nonbenzodiazepine hypnotic prescription at initial CCRC visit, \% & $13.2(7)$ & $5.1(3)$ & 0.187 \\
\hline Global trauma-related and depression symptoms & & & 0.009 \\
\hline Trauma-related and depression symptoms & $5.7(3)$ & $39.0(23)$ & \\
\hline Trauma-related symptoms, no depression symptoms & I5.I (8) & $25.4(15)$ & \\
\hline Depression symptoms, no trauma-related symptoms & $13.2(7)$ & $10.2(6)$ & \\
\hline No trauma-related or depression symptoms, history of depression ${ }^{\mathrm{b}}$ & $26.4(14)$ & $10.2(6)$ & \\
\hline No trauma-related or depression symptoms, no history of depression & $39.6(21)$ & $15.2(9)$ & \\
\hline
\end{tabular}

Notes: Continuous variables were expressed as average (SD). Categorical variables were expressed as \% (N). P-values are from comparisons between those with no changes in sleep and changes in sleep groups. Patients who were on any dose of a tricyclic antidepressant, serotonin reuptake inhibitor, serotonin-norepinephrine reuptake inhibitor, noradrenergic and specific serotonergic antidepressant (eg, mirtazapine), or norepinephrine and dopaminergic reuptake inhibitor (eg, bupropion) were considered to be on an antidepressant. Nonbenzodiazepine hypnotics were defined as drugs with effects similar to benzodiazepines, eg, zolpidem, zopiclone, eszopiclone, and zaleplon. Depression symptoms were defined as patients who indicated moderate to severe depressive symptoms (Geriatric Depression Scale-30 $\geq 20$ or Patient Health Questionnaire-9 $\geq 10$ ) at the initial CCRC visit prior to treatment in the CCRC. Trauma-related symptoms were defined as patients who endorsed having at least one of the following: nightmares, flashback or stressful memory of ICU stay, jumpiness (easily frightened or startled by sudden movements), or fear or place of situations that remind people of their ICU stay. aHospital stay with sentinel ICU stay resulting in CCRC referral. ${ }^{\circ}$ History of depression was defined as a diagnosis of depression based on informant report or chart diagnosis of depression. Fisher's exact tests were used to compare categorical outcomes for the two groups. Wilcoxon Rank-Sum tests were used to compare continuous outcomes for the two groups. Psychiatric and sleep disorders were defined as any relevant ICD-9 or ICD-I0 diagnoses by chart review within the 365 days prior to ICU admission.

Abbreviations: CCRC, Critical Care Recovery Center; CNS, central nervous disease; ICU, intensive care unit; MMSE, Mini-Mental State Examination.

half had at least one current trauma-related symptom $(43.8 \%$, $\mathrm{N}=49)$. More than half $(52.7 \%, \mathrm{~N}=59)$ reported sleep disturbances after their ICU stay.

Patients with sleep disturbances were younger, more likely to have current depressive symptoms, and more likely to have current trauma-related symptoms. Surprisingly, patients with alcohol use disorder and respiratory failure were less likely to have sleep disturbances. There were no differences in antidepressant or benzodiazepine/nonbenzodiazepine hypnotic use between patients with and without sleep disturbances. There were no differences in pre-existing psychiatric or sleep disorders between patients with and without sleep disturbances (Table 1). In a logistic regression analysis, the combination of trauma-related and depression symptoms (OR 16.66, 95\% CI 2.89-96.00) and trauma-related symptoms alone (OR 4.59, 95\% CI 1.11-18.88) were most closely 
Table 2 Psychiatric symptoms and sleep disturbances

\begin{tabular}{|l|l|l|}
\hline Demographic and clinical characteristics & $\begin{array}{l}\text { Sleep disturbances } \\
\text { OR (95\% CI) }\end{array}$ \\
\hline Age, years & $0.97(0.93-1.02)$ \\
\hline Hypertension & $0.96(0.29-3.21)$ \\
\hline Respiratory failure & $0.15(0.03-0.85)$ \\
\hline Antidepressant prescription at initial visit & $\mathrm{I} .10(0.33-3.65)$ \\
\hline Alcohol use disorder (current or previous) & $0.29(0.10-0.88)$ \\
\hline Global trauma-related and depression symptoms & & 0.952 \\
\hline Trauma-related and depression symptoms & $\mathrm{I} 6.66(2.89-96.00)$ \\
\hline Trauma-related symptoms, no depression symptoms & $4.59(\mathrm{I} . \mathrm{II}-\mathrm{I} 8.88)$ \\
\hline Depression symptoms, no trauma-related symptoms & $\mathrm{I} .35(0.23-7.83)$ \\
\hline No trauma-related or depression symptoms, history of depression & $0.95(0.23-3.89)$ \\
\hline No trauma-related or depression symptoms, no history of depression & $\mathrm{I} .00($ reference) \\
\hline
\end{tabular}

Notes: $\mathrm{OR}$ and $95 \% \mathrm{Cl}$ were calculated using logistic regression models. Models controlled for covariates $P<0.1$.

associated with a higher likelihood of sleep disturbances. When trauma-related symptoms were excluded, depression symptoms were no longer associated with sleep disturbances (OR 1.35, 95\% CI 0.23-7.83) (Table 2).

\section{Discussion}

The 2018 clinical practice guidelines for pain, agitation/ sedation, delirium, immobility, and sleep recognize the importance of addressing evidence gaps in sleep of critically ill patients. ${ }^{24}$ Our study suggests that trauma-related and depression symptoms are significant risk factors for sleep disturbances. It also suggests older patients are less prone to report subjective sleep disturbances, similar to prior studies suggesting older patients better tolerate chronic sleep deprivation. ${ }^{25,26}$ More than half of our post-ICU population reported sleep disturbances, consistent with previous studies. ${ }^{4}$ It was a diverse group with over $40 \%$ of the patients being African-American. They survived significant critical illness as the majority had respiratory failure requiring mechanical ventilation. Only $7.1 \%$ of patients had sleep disorders and $13.4 \%$ had psychiatric disorders prior to ICU admission. There were no differences in post-ICU sleep disturbances in these groups (Table 1).

Our study is consistent with prior studies showing a strong correlation between psychiatric symptoms and post-ICU sleep disturbances. Mental health symptoms were noted to be independently associated with poorer sleep quality in ICU survivors 6 months after discharge by McKinley et al. ${ }^{12}$ About 1 in 5 ICU survivors suffer from clinically significant PTSD symptoms and over one in four suffer from clinically significant depression. ${ }^{27,28}$ Our findings that post-ICU trauma-related symptoms alone and both trauma-related and depression symptoms were associated with post-ICU sleep disturbances suggest that psychiatric symptoms are a significant risk factor for post-ICU sleep disturbances. Given the lack of correlation between pre-ICU psychiatric diagnoses and post-ICU sleep disturbances in the CCRC cohort, this study suggests that ICU hospitalization may play a key role in the evolution of post-ICU psychiatric symptoms, particularly those related to trauma, and eventually, post-ICU sleep disturbances.

ICU survivors with sleep disturbances suffer from impaired Health-Related Quality of Life (HRQoL) ${ }^{4,5}$ and prior studies have tied psychological distress specifically to lower quality of life in these patients. ${ }^{10,29}$ The significant contribution of psychiatric morbidity to sleep disturbances and HRQoL in ICU survivors should be considered for targeted interventions while developing sleep-improvement programs. Our data contribute to the growing and needed epidemiologic basis of post-ICU morbidity and provide novel insights into psychiatric co-morbidities and sleep disturbances.

The finding of low sleep disturbances in patients with higher rates of respiratory failure and alcohol use disorder in this study is surprising, which differs from earlier findings. ${ }^{4,30}$ While the number of patients with alcohol use disorder in each group was low, many of the patients enrolled had respiratory failure. Our diverse, urban, single-center study may not be representative of a typical medical ICU regarding psychiatric conditions, alcohol use disorder, and other demographic and comorbid criteria. Therefore, these findings will need to be examined in more detail in future studies.

\section{Limitations}

This study had several limitations. First, the sample size of ICU survivors is fairly small and from a single site. Second, there were no objective data available to confirm the patients' subjective sleep complaints (eg, polysomnogram, actigra- 
phy) and the subjective questionnaire is not a validated one. Third, their exposure to non-pharmacologic treatments, eg, depression-focused psychotherapies, was unknown. Fourth, the possibility of selection bias was introduced without control of the variables measured in this study for the 3 months prior to their clinic visit. Fifth, although the questionnaire probed for trauma and stressor-related symptoms, they did not comprehensively cover all the PTSD symptoms.

\section{Conclusion}

ICU survivors who had active psychiatric symptoms were more likely to have subjective sleep disturbances. Future work will need to confirm these findings in larger, multisite longitudinal studies with validated sleep questionnaires in addition to objective measurements such as polysomnography or actigraphy. They will also need to better characterize psychiatric symptoms using DSM-5 criteria for specific diagnoses, and clarify further the relationships between sleep, psychotropic treatment, and psychotherapy in the ICU survivor population.

\section{Acknowledgment}

The Critical Care Recovery Center (CCRC) is supported by Eskenazi Health Services. This study was funded by Indiana Clinical and Translational Sciences Institute (NCATS UL1TR001108) (Project Development Team).

\section{Disclosure}

SW is supported by National Institute on Aging (NIA) 2P30AG010133, and National Center for Advancing Translational Science (NCATS) UL1TR001108 (Project Development Team). AJP is supported by National Heart, Lung, and Blood Institute (NHLBI) R01-HL131730 and NIA R01-AG055391. SG is supported by National Institutes of Health (NIH) P30AG010133. MAB is supported by grants R01 AG040220-05 and R01 AG030618-05A1, NIA, grant P30 HS024384-02, Agency for Healthcare Research and Quality, and grant 1 L1 CMS331444-02-00, Centers for Medicare and Medicaid Services. BAK is supported by NHLBI R01-HL131730 and NIA R01-AG055391. The authors report no other conflicts of interest in this work.

\section{References}

1. Elliott R, Mckinley S, Cistulli P. The quality and duration of sleep in the intensive care setting: an integrative review. Int J Nurs Stud. 2011;48(3):384-400.

2. Little A, Ethier C, Ayas N, et al. A patient survey of sleep quality in the intensive care unit. Minerva Anestesiol. 2012;78(4):406-414.

3. Pisani MA, Friese RS, Gehlbach BK, Schwab RJ, Weinhouse GL, Jones SF. Sleep in the intensive care unit. Am J Respir Crit Care Med. 2015;191(7):731-738.
4. Altman MT, Knauert MP, Pisani MA. Sleep disturbance after hospitalization and critical illness: a systematic review. Ann Am Thorac Soc. 2017;14(9):1457-1468.

5. Orwelius L, Nordlund A, Nordlund P, Edéll-Gustafsson U, Sjöberg F. Prevalence of sleep disturbances and long-term reduced health-related quality of life after critical care: a prospective multicenter cohort study. Crit Care. 2008;12(4):R97.

6. Boyko Y, Jennum P, Toft P. Sleep quality and circadian rhythm disruption in the intensive care unit: a review. Nat Sci Sleep. 2017;9: 277-284.

7. Kamdar BB, Needham DM, Collop NA. Sleep deprivation in critical illness: its role in physical and psychological recovery. J Intensive Care Med. 2012;27(2):97-111.

8. Krystal AD. Psychiatric disorders and sleep. Neurol Clin. 2012;30(4): 1389-1413.

9. Manber R, Edinger JD, Gress JL, et al. Cognitive behavioral therapy for insomnia enhances Clinical and Translational Sciences Institute depression outcome in patients with comorbid major depressive disorder and insomnia. Sleep. 2008;31(4):489-495.

10. Mckinley S, Aitken LM, Alison JA, et al. Sleep and other factors associated with mental health and psychological distress after intensive care for critical illness. Intensive Care Med. 2012;38(4):627-633.

11. Mckinley S, Fien M, Elliott R, Elliott D. Sleep and psychological health during early recovery from critical illness: an observational study. $J$ Psychosom Res. 2013;75(6):539-545.

12. Parsons EC, Hough CL, Vitiello MV, Zatzick D, Davydow DS. Insomnia is associated with quality of life impairment in medical-surgical intensive care unit survivors. Heart Lung. 2015;44(2):89-94.

13. Solverson KJ, Easton PA, Doig CJ. Assessment of sleep quality post-hospital discharge in survivors of critical illness. Respir Med. 2016;114:97-102.

14. Ely EW, Shintani A, Truman B, et al. Delirium as a predictor of mortality in mechanically ventilated patients in the intensive care unit. JAMA. 2004;291(14):1753-1762.

15. Mukherjee S, Patel SR, Kales SN, et al. An official American Thoracic Society statement: the importance of healthy sleep. recommendations and future priorities. Am J Respir Crit Care Med. 2015;191(12): 1450-1458.

16. Parthasarathy S, Vasquez MM, Halonen M, et al. Persistent insomnia is associated with mortality risk. Am J Med. 2015;128(3):268-275.

17. Schmid SM, Hallschmid M, Jauch-Chara K, et al. Sleep loss alters basal metabolic hormone secretion and modulates the dynamic counterregulatory response to hypoglycemia. J Clin Endocrinol Metab. 2007;92(8):3044-3051.

18. Spiegel K, Sheridan JF, van Cauter E. Effect of sleep deprivation on response to immunization. JAMA. 2002;288(12):1471-1472.

19. Barnato AE, Albert SM, Angus DC, Lave JR, Degenholtz HB. Disability among elderly survivors of mechanical ventilation. Am J Respir Crit Care Med. 2011;183(8):1037-1042.

20. Pandharipande PP, Girard TD, Jackson JC, et al. Long-term cognitive impairment after critical illness. $N$ Engl J Med. 2013;369(14): 1306-1316.

21. Salluh JIF, Wang H, Schneider EB, et al. Outcome of delirium in critically ill patients: systematic review and meta-analysis. $B M J$. 2015;350:h2538.

22. American Psychiatric Association. Diagnostic and Statistical Manual of Mental Disorders. 5th ed. Washington D.C.; 2013. Available from: http:// dx.doi.org/10.1176/appi.books.9780890425596. Accessed December 16, 2018.

23. Bursac Z, Gauss CH, Williams DK, Hosmer DW. Purposeful selection of variables in logistic regression. Source Code Biol Med. 2008;3: 17.

24. Devlin JW, Skrobik Y, Gélinas C, et al. Clinical practice guidelines for the prevention and management of pain, Agitation/Sedation, delirium, immobility, and sleep disruption in adult patients in the ICU. Crit Care Med. 2018;46(9):e825-e873. 
25. Duffy JF, Willson HJ, Wang W, Czeisler CA. Healthy older adults better tolerate sleep deprivation than young adults. J Am Geriatr Soc. 2009;57(7):1245-1251.

26. Zitting KM, Münch MY, Cain SW, et al. Young adults are more vulnerable to chronic sleep deficiency and recurrent circadian disruption than older adults. Sci Rep. 2018;8(1):11052.

27. Davydow DS, Gifford JM, Desai SV, Needham DM, Bienvenu OJ. Posttraumatic stress disorder in general intensive care unit survivors: a systematic review. Gen Hosp Psychiatry. 2008;30(5):421-434.
28. Davydow DS, Gifford JM, Desai SV, Bienvenu OJ, Needham DM. Depression in general intensive care unit survivors: a systematic review. Intensive Care Med. 2009;35(5):796-809.

29. Mckinley S, Fien M, Elliott R, Elliott D. Health-related quality of life and associated factors in intensive care unit survivors 6 months after discharge. Am J Crit Care. 2016;25(1):52-58.

30. Stein MD, Friedmann PD. Disturbed sleep and its relationship to alcohol use. Substance Abuse. 2006;26(1):1-13.

\section{Publish your work in this journal}

The International Journal of General Medicine is an international, peer-reviewed open-access journal that focuses on general and internal medicine, pathogenesis, epidemiology, diagnosis, monitoring and treatment protocols. The journal is characterized by the rapid reporting of reviews, original research and clinical studies across all disease areas.
The manuscript management system is completely online and includes a very quick and fair peer-review system, which is all easy to use. Visit http://www.dovepress.com/testimonials.php to read real quotes from published authors.

Submit your manuscript here: https://www.dovepress.com/international-journal-of-general-medicine-journal 